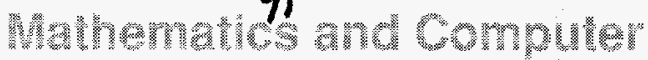

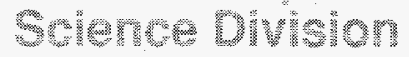

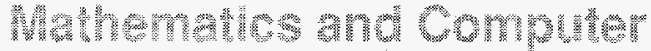

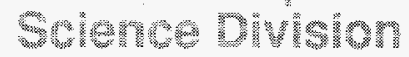

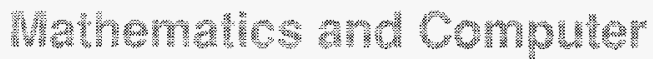

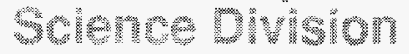

Wa hemolics and Comoutur

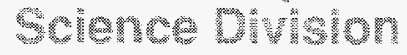

Mathematics and Computer

Science Division

Mathematics and Computer

Science Division

Mathematics and Computer

Science Division

Whametics and Gompurer

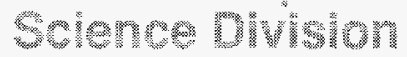

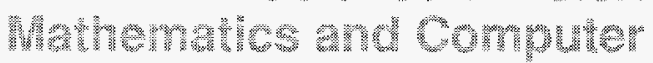

sclence Mivisum

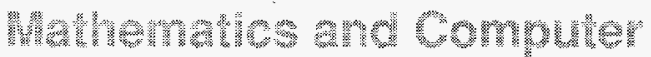

somen Dhiston

Whathendew and Compurer

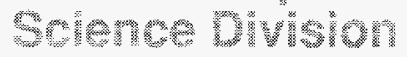

Whathematics and compuler

s chance Divisum

Whanomics and Communt

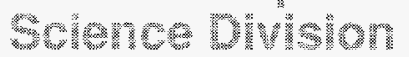

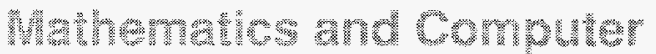

science Düumon

Wathemencs anc Compluck

solonct Division

Whathemsics and Compurer

sulvmes Divish

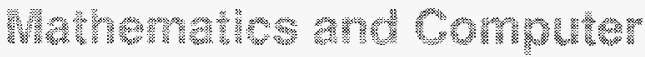

Solence division

\section{BlockSolve95 Users Manual: Scalable Library Software for the Parallel Solution of Sparse Linear Systems}

by M. T. Jones and P. E. Plassmann

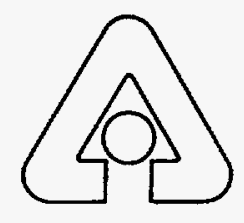

Argonne National Laboratory, Argonne, Illinois 60439

operated by The University of Chicago

for the United States Department of Energy under Contract W-31-109-Eng-38

Mathematics and Computer

Sclence division

Matherratics and Computer

Scletice Division

Mathematics and Computer

Science Diviston

Mathematios and Computer

Science Division 
Argonne National Laboratory, with facilities in the states of Illinois and Idaho, is owned by the United States government, and operated by The University of Chicago under the provisions of a contract with the Department of Energy.

\section{DISCLAIMER}

This report was prepared as an account of work sponsored by an agency of the United States Government. Neither the United States Government nor any agency thereof, nor any of their employees, makes any warranty, express or implied, or assumes any legal liability or responsibility for the accuracy, completeness, or usefulness of any information, apparatus, product, or process disclosed, or represents that its use would not infringe privately owned rights. Reference herein to any specific commercial product, process, or service by trade name, trademark, manufacturer, or otherwise, does not necessarily constitute or imply its endorsement, recommendation, or favoring by the United States Government or any agency thereof. The views and opinions of authors expressed herein do not necessarily state or reflect those of the United States Government or any agency thereof.

Reproduced from the best available copy.

Available to DOE and DOE contractors from the Office of Scientific and Technical Information

P.O. Box 62

Oak Ridge, TN 37831

Prices available from (423) 576-8401

Available to the public from the National Technical Information Service

U.S. Department of Commerce 5285 Port Royal Road

Springfield. VA 22161 
Distribution Category:

Mathematics and

Computer Science (UC-405)

\section{ARGONNE NATIONAL LABORATORY}

9700 South Cass Avenue

Argonne, IL 60439

ANL-95/48

\section{BlockSolve95 Users Manual: \\ Scalable Library Software for the \\ Parallel Solution of Sparse Linear Systems}

by

Mark T. Jones* and Paul E. Plassmann

Mathematics and Computer Science Division

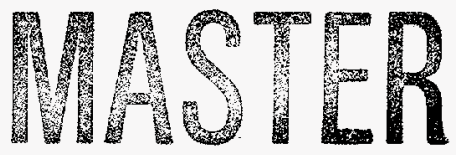

December 1995

(Revised June 1997)

*Address: Dept. of Computer Science, The University of Tennessee, 107 Ayres Hall, Knoxville, TN 37996-1301

This work was supported by the Mathematical, Information, and Computational Sciences Division subprogram of the Office of Computational and Technology Research, U.S. Department of Energy, under Contract W-31-109-Eng-38. The first author was supported in part by a 1994-95 University of Tennessee Professional Development award and NSF Grants ASC-9501583 and ASC-9411394. 


\section{DISCLAMMER}

Portions of this document may be illegible in electronic image products. Images are produced from the best available original document. 


\section{Contents}

$\begin{array}{ll}\text { Abstract } & 1\end{array}$

1 Introduction 2

2 Algorithm Descriptions 4

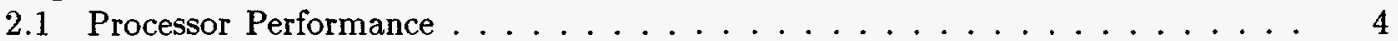

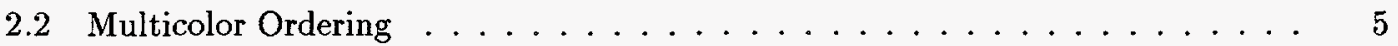

2.3 Communication Efficiency $\ldots \ldots \ldots \ldots \ldots \ldots$

3 The BlockSolve95 Computing Environment 8

3.1 Initializing and Finalizing the BlockSolve95 Environment . . . . . . . . 8

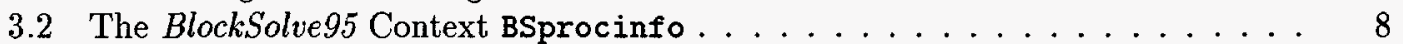

3.2.1 Parallel Environment Options . . . . . . . . . . . . . . . 8

3.2 .2 Factorization Options .................. 9

3.2 .3 Solver Options . . . . . . . . . . . . . . . . . 11

4 The User Matrix Data Structure BSspmat 12

4.1 The Easy Way to Convert Your Data to BSspmat . . . . . . . . . . . . . . . 12

4.2 The Advanced Way to Convert Matrix Data to BSspmat . . . . . . . . . . . 13

5 Manipulating and Solving Sparse Systems 16

5.1 Manipulation and Setup . . . . . . . . . . . . . . . . . . 16

5.1 .1 Symmetric and Nonsymmetric Matrices . . . . . . . . . . . . . 16

5.1.2 Fast Reorderings for Different Matrix Nonzero Values . . . . . . . . . 16

5.1.3 Diagonal Scaling of the Linear System . . . . . . . . . . . . . . 17

5.1.4 The Communication Data Structure BScomm . . . . . . . . . . . . 17

5.1.5 Computing an Incomplete Factorization . . . . . . . . . . . . . . 18

5.1 .6 What to Do If the Factorization Fails . . . . . . . . . . . . 18

5.2 Solving the Linear System . . . . . . . . . . . . . . . . . . . . . 19

5.3 Freeing Matrices . . . . . . . . . . . . . . . . . . . . 20

6 Error Checking, Flop Counting, Message Passing, and the BLAS 21

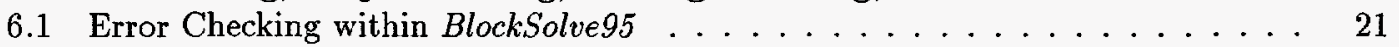

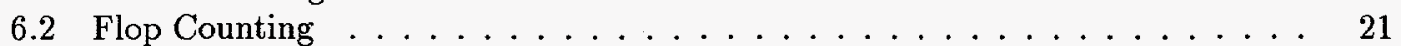

6.3 Matrix Statistics . . . . . . . . . . . . . . . . . . . 22

6.4 Blocking and Nonblocking Messages . . . . . . . . . . . . . . . . 22

6.5 MPI Communicators and Message Number Conflicts . . . . . . . . . . . . . 22

6.6 Inline Macros for the BLAS . . . . . . . . . . . . . . . . . . 23

7 Installation $\quad \mathbf{2 4}$

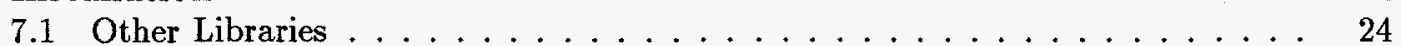

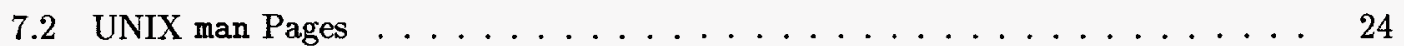

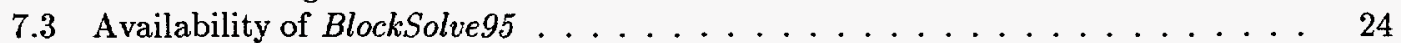

7.4 Differences between Previous BlockSolve Versions . . . . . . . . . . . . 25

8 Example Programs $\quad 26$

9 Limitations and Future Plans $\quad 29$

$\begin{array}{ll}10 \text { Related Software } & 30\end{array}$ 
References

Subject Index

Function Index 


\title{
BlockSolve95 Users Manual: \\ Scalable Library Software for the \\ Parallel Solution of Sparse Linear Systems
}

by

Mark T. Jones and Paul E. Plassmann

\begin{abstract}
BlockSolve95 is a software library for solving large, sparse systems of linear equations on massively parallel computers or networks of workstations. The matrices must be symmetric in structure; however, the matrix nonzero values may be either symmetric or nonsymmetric. The nonzeros must be real valued. BlockSolve 95 uses a message-passing paradigm and achieves portability through the use of the MPI message-passing standard. Emphasis has been placed on achieving both good processor performance through the use of higher-level BLAS and scalability through the use of advanced algorithms. This report gives detailed instructions on the use of BlockSolve95 and descriptions of a number of program examples that can be used as templates for application programs.
\end{abstract}




\section{Introduction}

BlockSolve95 is a scalable parallel software library primarily intended for the solution of sparse linear systems that arise from physical models, especially problems involving multiple degrees of freedom at each node. For example, when the finite element method is used to solve practical problems in structural engineering, each node typically has two to five degrees of freedom; BlockSolve95 is designed to take advantage of problems with this type of local structure. BlockSolve95 is also reasonably efficient for problems that have only one degree of freedom associated with each node, such as the three-dimensional Poisson problem. BlockSolve95 is general purpose; we require only that the matrices be sparse and symmetric in structure (but not necessarily in value). ${ }^{1}$

BlockSolve95 has incorporated several features that allow efficient performance on diverse parallel architectures. We summarize these features below:

- Every aspect of the linear system solution-the computation of a matrix ordering, the renumbering, the computation of the preconditioner, and the iterative solution-is done in parallel.

- BlockSolve95 runs on a variety of parallel architectures and can easily be ported to others. The MPI message-passing standard $[4,13]$ is used to achieve portability across architectures. Machines on which BlockSolve95 has been tested include the IBM SP series, the Cray T3D and T3E, the SGI Cray Origin2000, the HP-Convex Exemplar, the Intel Paragon, and networks of Sun, SGI, DEC alpha, and HP workstations. The bmake directory contains contains a complete set of the machine-dependent makefiles for the architectures currently supported.

- The software uses an efficient implementation of the parallel coloring algorithm described in [9] that allows for the efficient computation of matrix orderings and the scalable performance of the linear solver described below.

- The software is designed to solve linear systems whose sizes are the same order as the total amount of memory available on a parallel machine. For example, Table 1 shows how effectively BlockSolve95 uses the available memory on two different architectures for the high-temperature superconductor modeling application described in [8]. Note that some of each processor's memory is used by the operating system and program executable (e.g., approximately 4 MBytes and 1 MByte, respectively, on the Intel DELTA). For this application an unpermuted version of the matrix is maintained in addition to the memory required by BlockSolve95. Moreover, the memory required to store only the indices and the double-precision values for $1.0 \times 10^{8}$ nonzeros is 1.2 GBytes.

Table 1: Effective use of total memory by BlockSolve95

\begin{tabular}{|l|c|c|l|l|}
\hline Machine & $\begin{array}{l}\text { Number of } \\
\text { Processors }\end{array}$ & $\begin{array}{c}\text { Number of } \\
\text { Matrix } \\
\text { Nonzeros }\end{array}$ & $\begin{array}{l}\text { Memory per } \\
\text { Processor } \\
\text { (Usable } \\
\text { Memory) }\end{array}$ & $\begin{array}{l}\text { Total } \\
\text { Memory } \\
\text { (Usable } \\
\text { Memory) }\end{array}$ \\
\hline $\begin{array}{l}\text { Intel } \\
\text { DELTA }\end{array}$ & 512 & $1.9 \times 10^{8}$ & $\begin{array}{l}16 \text { MBytes } \\
(11 \text { MBytes) }\end{array}$ & $\begin{array}{l}8 \text { GBytes } \\
\text { (5.6 MBytes) }\end{array}$ \\
\hline IBM SP1 & 128 & $5.1 \times 10^{8}$ & 128 MBytes & 16 GBytes \\
\hline
\end{tabular}

\footnotetext{
${ }^{1}$ Whenever we use the term nonsymmetric with respect to BlockSolve95, we mean symmetric in structure and nonsymmetric in value. Thus, BlockSolve95 handles most matrices arising in finiteelement calculations; however, it cannot handle general nonsymmetric matrices.
} 
- The software is designed to achieve scalable performance. With a constant number of unknowns per processor, a matrix nonzero structure resulting from local graph connections (the usual case with any finite-element mesh), and a reasonable partitioning of vertices to processors, the average processor performance is roughly constant. For example, for the piezoelectric crystal modeling application described in [7], the average processor performance on the Intel DELTA varied from 4.16 MFlops to 3.83 MFlops when the number of processors was increased from 128 to 512 .

- The software is designed to use Level 2 and Level 3 dense BLAS (the Basic Linear Algebra Subroutines) to achieve efficient use of processors on different architectures. For example, Table 2 shows the average processor performances for the application described in [8]. Note that a reasonable percentage of the LINPACK benchmark performance is achieved for a sparse matrix calculation on these very different processor architectures.

Table 2: Efficient use of different processor architectures by BlockSolve95

\begin{tabular}{|l||l|l|l|l|}
\hline Machine & $\begin{array}{l}\text { Processor } \\
\text { Architecture }\end{array}$ & $\begin{array}{l}\text { Number } \\
\text { of } \\
\text { Processors }\end{array}$ & $\begin{array}{l}\text { Avg. Proc. } \\
\text { Performance } \\
\text { Achieved }\end{array}$ & $\begin{array}{l}\text { Total } \\
\text { Performance }\end{array}$ \\
\hline Intel DELTA & Intel i860 & 512 & 8.3 MFlops & 4.26 GFlops \\
\hline IBM SP1 & RS/6000-370 & 128 & 20.5 MFlops & 2.62 GFlops \\
\hline
\end{tabular}

- The software is a general-purpose sparse solver. Hence, BlockSolve95 is effective also on problems arising from unstructured meshes. For example, a total performance of 2.2 GFlops was achieved on 512 processors of the Intel DELTA for an adaptive mesh calculation using high-order shell elements in a three-dimensional geometry [10].

- The software requires minimal input. You must give BlockSolve95 the matrix nonzeros and global indices of rows corresponding to the unknowns assigned to each processor and the mapping functions to translate between global and local indexing.

- BlockSolve95 is designed to be most effective within real application codes. In our experience, most application codes must solve the same linear systems with several different right-hand sides and/or solve linear systems with the same structure, but different matrix values, multiple times. BlockSolve95 has, therefore, been designed to work well in this situation.

The remainder of this manual is organized as follows. We begin in $\S 2$ with a brief description of the algorithms used in BlockSolve95. In $\S 3, \S 4$, and $\S 5$ we present the routines required to set up the context and matrix data structures and solve linear systems with BlockSolve95. In $\$ 6$ we discuss a number of details dealing with flop counting, BLAS performance, message-passing options, and other issues that arise in tuning the performance of an application. The installation and availability of BlockSolve 95 are discussed in $\S 7$, and in $\S 8$ we describe the program examples that are included with this version. Finally, in $\S 9$ we discuss related software and plans for future versions of the code.

This document is intended to be used primarily as a reference. If you are a new user, we recommend using the BlockSolve95 examples as templates for your application codes. 


\section{Algorithm Descriptions}

The primary algorithmic components of BlockSolve95 are

- the computation of a matrix ordering allowing for the scalable inversion of the triangular systems arising from incomplete matrix factorizations;

- the automatic extraction of clique and identical node (i-node) information from unstructured systems that allows for the use of higher-level BLAS; and

- the computation of incomplete sparse matrix factorizations for use as preconditioners in the iterative solution of linear systems.

The matrix-ordering algorithms are used to ensure that, for many problems, the performance per processor remains roughly constant as the problem size and number of processors are increased. Computational efficiency is obtained through the use of higher-level BLAS, efficient matrix storage schemes, and precomputed communication data structures. For symmetric matrices, incomplete Cholesky factorizations may be computed; and for general matrices, incomplete LU factorizations may be obtained. These preconditioners are designed so that their application is both efficient and as scalable as possible.

In addition, BlockSolve 95 contains implementations of several well-known iterative methods. A preconditioned conjugate gradient method can be used for symmetric, positive definite systems. For symmetric indefinite systems, the preconditioned SYMMLQ algorithm may be used. $^{2}$ For nonsymmetric systems, the GMRES method may be used. In addition, the PETSc software package [1] contains an interface to the BlockSolve95 preconditioners; this interface may be used if other iterative methods are required. For basic information on iterative methods, see [2].

You can select from a number of different preconditioners. The first is a simple diagonal scaling of the matrix, which can be used by itself or in conjunction with another preconditioner. The other preconditioning options are incomplete Cholesky or LU factorization, SSOR $(\omega=1)$, and block Jacobi (where the blocks are the cliques of the graph associated with the sparse matrix). Perhaps the most generally applicable selection is the incomplete factorization with diagonal scaling. ${ }^{3}$ The incomplete factorization is the algorithm for which BlockSolve95 was designed; this approach has proved useful in a wide variety of practical problems.

BlockSolve95 does not partition a matrix across the processors. Instead, BlockSolve95 assumes that the given partitioning is a good one. As such, its performance may be limited by the quality of the partitioning. We assume that the right-hand side and the solution vector are partitioned in the same manner as the rows of the sparse matrix. See [5] for more information on partitioning heuristics.

We achieve parallelism in the conjugate gradient, SYMMLQ, and GMRES implementations by partitioning the vectors used in these algorithms in the same manner that the rows of the matrix are partitioned across the processors. For these algorithms it is (for the most part) a simple matter of executing inner products and daxpy operations in parallel.

\subsection{Processor Performance}

To achieve good performance on each node, we reorder the matrix and use a layered data structure to allow the use of the higher-level dense BLAS. This reordering is particularly important

\footnotetext{
${ }^{2}$ The SYMMLQ algorithm requires a positive definite preconditioner, and this requirement can be a serious limitation if the matrix being solved is very indefinite. By "very indefinite," we mean that the matrix has many negative and many positive eigenvalues.

${ }^{3}$ Two possible exceptions to this recommendation are (1) if the matrix has no or very small cliques and identical nodes (in which case the factorization may be. very slow) and (2) if the space for the incomplete factorization is not available.
} 
on machines that use high-performance RISC chips on which good performance can be achieved only by using such operations. The reordering of the matrices is based on the identification of two structures that commonly arise in the graph associated with the matrix nonzeros: identical nodes and cliques.

Identical nodes (i-nodes) typically exist when multiple degrees of freedom are associated with each vertex in the graph. Cliques are found in many graphs associated with sparse matrices, but cliques typically are found in graphs where multiple degrees of freedom are associated with each vertex and the local connectivity of the graph is large. For example, if one uses a second-order, three-dimensional finite element in a typical structural engineering problem (with three degrees of freedom per vertex), clique sizes of up to 81 can be found. In general, the larger the cliques or identical nodes, the better the performance. This technique has been used with great success in direct matrix factorization methods.

We illustrate these structures in Figure 1 where we depict a subsection of a graph that would arise from a two-dimensional, bilinear, multicomponent finite-element model with three degrees of freedom per discretization point. We illustrate the three degrees of freedom by the three dots at each node point; the linear, quadrilateral elements imply that the twelve degrees of freedom sharing the four node points of each face are completely connected. In the figure we show edges only between the nodes, these edges represent the complete interconnection of all the vertices on each element or face.

The dashed lines in the figure represent a geometric partitioning of the grid; we assume that the vertices in the central region are all assigned to one processor. We note that the adjacency structures of the vertices at the same geometric node (i.e., the nonzero structure of the associated variables) are identical. BlockSolve95 takes advantage of these so-called i-nodes by maintaining only one copy of the indexing for each set of identical rows and by storing nonzeros for these rows in a dense matrix form that can be used with the BLAS.

A second data structure layer is determined by the clique structure of the graph. Recall that a clique is a completely connected subgraph. In the upper right of Figure 1, the grouping of vertices by the dotted lines partitions the graph into cliques. BlockSolve95 associates a "supernode" with each clique and orders the unknowns associated with each clique consecutively. Based on this ordering, the submatrix corresponding to the unknowns in the same clique corresponds to a dense matrix. In the sparse matrix, these dense matrices manifest themselves as dense blocks on the matrix diagonal. The BlockSolve95 data structures explicitly store these dense blocks to allow for greater efficiency in using the BLAS.

\subsection{Multicolor Ordering}

Following the graph reductions that are accomplished after the identification of i-nodes and cliques in the sparse matrix structure, BlockSolve95 must construct a matrix ordering based on the reduced graph, illustrated at the bottom of Figure 1. To achieve scalable parallel performance in the incomplete factorization and SSOR preconditioners, BlockSolve95 colors the reduced graph using a parallel coloring heuristic [9]. The graph reduction and coloring phases of the computation are efficient and typically require a small amount of time relative to the incomplete matrix factorization routine.

The advantage of using a graph coloring is that the number of colors required is essentially a local property of the graph; thus, the number of colors is roughly independent of the number processors used for a fixed discretization scheme. This fact allows for the scalable performance of the BlockSolve95 package [11]. The trade-off for this scalability is that these multicolor orderings may not be the optimal orderings to choose for minimizing the number of iterations required for the iterative solver; the convergence of the iterative solver with respect to different orderings is highly problem dependent. However, the combination of coloring a general symmetric sparse matrix and the incomplete Cholesky algorithm has proved to be successful for solving large 


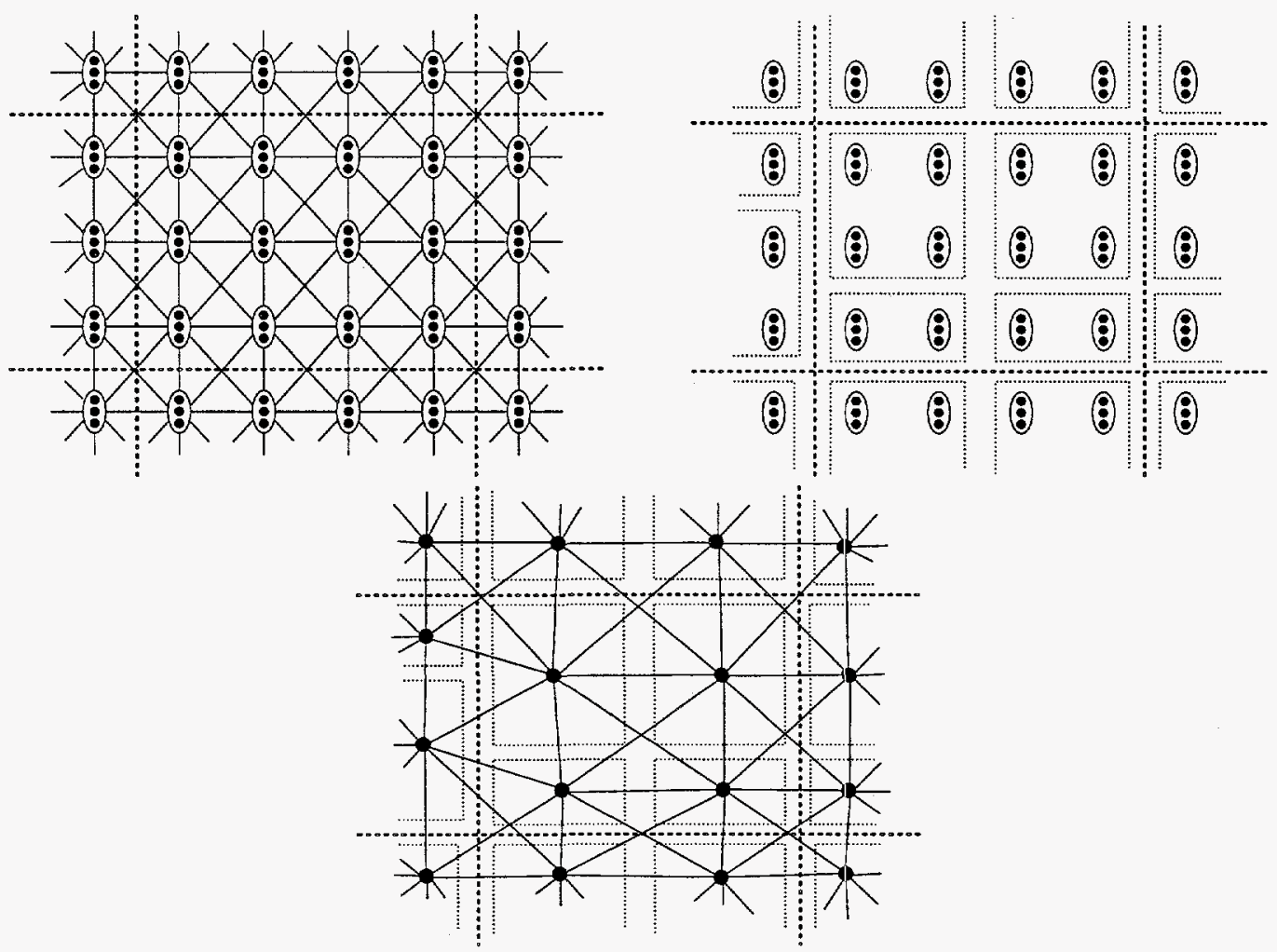

Figure 1: A subgraph generated by a two-dimensional, bilinear finite-element model with three degrees of freedom per discretization point. The geometric partition shown by the dotted lines yields an assignment of the vertices in the enclosed subregion to one processor. The upper right figure shows one possible decomposition of the graph into local cliques, and the lower figure shows the corresponding clique (or supernode) graph.

problems on scalable parallel architectures [7]. In [6] we address the issue of convergence of this combination of algorithms for the model problem.

\subsection{Communication Efficiency}

The computational kernel of the BlockSolve95 package is the solution of triangular matrix systems and triangular matrix multiplication. Triangular matrices of the same structure are solved and multiplied in parallel many times in the use of one of the iterative solvers. The key observation is that the required interprocessor communication in triangular system solve or multiply is the same every time; the only difference is in the floating-point values that are sent and received.

To take advantage of this repeated communication pattern, the BlockSolve95 routines in BMcomp_msg() are used to build reusable data structures. During each communication phase each processor needs to send and receive data from some subset of the processors (a small subset, if the partitioning is good). Each message to be sent is an array of floating-point values and an array of integer values (matrix indices). A key observation is that these integer values do not change; only the floating-point values change. 
Prior to any triangular matrix solution and multiplication, each processor declares (via function calls in BMcompmsg( $)$ ) the messages that it will be sending and receiving and specifies the integer index values associated with these messages. The routines in BMcompmsg() build data structures by assembling the declarations from each processor. In addition, the integer index values associated with the messages are sent to the appropriate processor and stored there; these values will not be sent in the future. Since the size of the required messages is now known, message buffers can be preallocated.

We currently perform no special communication pattern optimizations in BMcompmsg() although these routines have been set up for this possibility. The optimizations currently performed are

1. presending of integer values;

2. preallocation of message buffers, allowing "forced" messages; MPI_Irsend (), to be used; and

3. no requests are made between processors for non-local data (processors use local information to know what messages to expect and what to send).

For an example of the message setup process, see the routine BSsetup forward(). For an example of how the message data structures are used, see the routine BSforward(). 


\section{The BlockSolve95 Computing Environment}

In this section we describe the BlockSolve95 computing environment. BlockSolve95 has an initialization routine that must be called prior to any other BlockSolve95 routine and a finalization routine that must be called last. We also introduce the most important BlockSolve95 data structure-BSprocinfo-the BlockSolve95 context. All information about the parallel environment, preconditioner options, and settings for the iterative solvers is communicated to the BlockSolve95 package through the BSprocinfo context data structure.

\subsection{Initializing and Finalizing the BlockSolve95 Environment}

The first call to a BlockSolve95 routine must be the initialization routine BSinit(). The definition and arguments for this routine are as follows.

int BSinit(int *argc, char ***args);

The arguments argc and argv should be the command line arguments as delivered in all $\mathrm{C}$ and $\mathrm{C}++$ programs. If you have not already done so, this routine initializes MPI with a call to MPI_Init () and initializes any logging routines that have been specified (see $\$ 6$ for more information on logging within BlockSolve95).

The final call to a BlockSolve95 routine must be the routine BSfinalize().

int BSfinalize();

This routine calls MPI_Finalize() if BlockSolve95 initialized MPI.

\subsection{The BlockSolve95 Context BSprocinfo}

The BlockSolve95 context data structure, typedef BSprocinfo, contains all the information about the parallel environment, options for computing the preconditioner, and settings for the iterative solver. Before calling any BlockSolve95 routines, the user must first allocate a context of data type BSprocinfo for BlockSolve95 using the routine BScreate_ctx().

BSprocinfo *BScreate_ctx();

After the last BlockSolve95 routine has been called, the context should be freed by calling the routine-BSfree_ctx().

void BSfree_ctx(BSprocinfo *context);

After the call to BScreate_ctx(), you can then call one of many routines to modify the context. We provide default settings for the context that we think will, in general, provide the best performance, but you may benefit from changing some of the settings.

The context options can be broken into three broad categories: (1) the parallel environment, (2) options concerning the computation of preconditioners, and (3) settings for calls to an iterative solver. In the following three subsections we describe the possible options and give the routine names that modify the context.

\subsubsection{Parallel Environment Options}

The following options concern the general parallel environment in which BlockSolve95 operates. The settings and routines for changing them are as follows: 
- Processor Set: Definition of the processors that are participating in this call to BlockSolve95. If the number of processors participating is equal to the number of processors that are allocated to the user (this is the default case), this value should be set to MPI_COMM_WORLD. If, for example, you wish to work on different matrices on different sets of processors at the same time and perhaps later combine the answers, you must set the procset parameter accordingly. The program example grid2 solves two linear systems simultaneously on a partitioned set of processors. The default setting for this parameter is MPI_COMM_WORLD. To reset the value, call the routine BSctx_set_ps().

void BSctx_set_ps (BSprocinfo *context, ProcSet *ps);

You may wish to set the processor set to a duplicate communicator (using MPI_Comm_dup()) to ensure that there are no message conflicts.

- Processor id: The id number of this processor. The default setting is given by the routine MPI_Comm_rank(). To reset the value, call the routine BSctx_set_id().

void BSctx_set_id(BSprocinfo *context, int id);

- Number of processors: The number of processors that are calling BlockSolve95 with a portion of the matrix. The default setting is given by the routine MPI_Comr_size(). To reset the value, call the routine BSctx_set_np().

void BSctx_set_np(BSprocinfo *context, int np);

- Error checking: Simple error checking on the user's matrix structure and on some intermediate data structures. The error checking is not very time consuming and is probably a good option to use for the first few runs. The default setting is FaLSE (i.e., no error checking). To change this value, call the routine BSctx_set_err().

void BSctx_set_err(BSprocinfo *context, int err);

- Print information: Print information about the coloring, reordering, and linear system solution options. If set to TRUE this information is printed during execution. The default setting is FALSE. To change this value, call the routine BSctx_set_pr().

void BSctx_set_pr(BSprocinfo *context, int pr);

- Print logging information: Print logging information when the routine BSprint $\_\circ g()$ is called. If set to TRUE this information is printed, the default setting is FALSE. To change this value, call the routine BSctx_set_print_log().

void BSctx_set_print_log(BSprocinfo *context, int print);

- Print current context options: The current context options. To print information on how the context is currently set, call the function BSctx_print().

void BSctx_print(BSprocinfo *context);

\subsubsection{Factorization Options}

The following options concern the computation of incomplete factorizations by BlockSolve95. Calls to these routines must be made prior to calling BSmain_perm(). The settings and routines for changing them are as follows: 
- Maximum clique size: The maximum number of rows in a single clique. You may wish to limit this value if the cliques become too large and performance is impaired (an unlikely case in most applications and something that requires understanding the algorithms in BlockSolve95). The default setting is INT MAX, which is defined in the system include file limits.h. To change this value, call the routine BSctx_set_cs().

void BSctx_set_cs(BSprocinfo *context, int cs);

- Maximum identical node size: The maximum number of rows combined into an identical node. You may wish to limit this value if the i-nodes become too large and performance is impaired (an unlikely case in most applications and something that requires understanding the algorithms in BlockSolve95). The default setting is INT MAX. To change this value, call the routine BSctx_set_is().

void BSctx_set_is(BSprocinfo *context, int is);

- Type of local coloring: The type of local coloring heuristic used. There are two phases in obtaining a coloring of the matrix graph: a global phase in which the Jones/Plassmann algorithm is used and a local phase where either an incident degree ordering (IDO) coloring or a saturated degree ordering (SDO) coloring is used. In general, the SDO colorings are slightly better but take more time to find. The default setting is IDO. To change this value, call the routine BSctx_set_ct( ).

void BSctx_set_ct(BSprocinfo *context, int ct);

- Retain data structures: Information retained during the reordering process. The information saved (if the flag is set to TRUE) allows a fast reordering if a matrix with the same structure is to be reordered later. The default setting is FALSE. To change this value, call the routine BSctx_set_rt().

void BSctx_set_rt(BSprocinfo *context, int $r t)$;

- No clique/i-node reordering: No attempt to find cliques or i-nodes. This flag should be set to TRUE (i.e., no search for i-nodes or cliques will be made) when you know that the i-node or clique sizes will be 1 or very close to 1 (you may wish to experiment with this). The default setting is FALSE. To change this value, call the routine BSctx_set_si().

void BSctx_set_si(BSprocinfo *context,int si);

- Scale linear system: Scaling of the linear system. The default setting is TRUE. To change this value, BSctx_set_scaling() may be called.

void BSctx_set_scaling(BSprocinfo *context, int scale);

Note that if this function is set, the routine BSscale_diag() must be called to do the scaling. We recommend using the matrix diagonal for scaling so that the absolute value of the diagonal of the scaled system is set to one. 


\subsubsection{Solver Options}

The following options concern the solution of linear systems by BlockSolve95. Calls to these routines must be made prior to calling BSpar_solve() or BSpar_isolve(). The settings and routines for changing them are as follows:

- Maximum number of iterations: The maximum number of iterations allowed the iterative solver. The default setting is 100 . To change this value, call the routine BSctx_set_max_it().

void BSctx_set_max_it(BSprocinfo *context, int max_it)

- GMRES restart value: The maximum number of vectors allowed GMRES before restarting. The default setting is 20 . To change this value, call the routine BSctx_set_restart().

void BSctx_set_restart(BSprocinfo *context, int restart);

- Initial guess for iterative method: The initial vector used by the iterative method. If this flag is TRUE, the initial guess is the zero vector. If FALSE, the iterative method uses the vector passed to it. This option is useful if a good estimate of the solution is available. The default setting is TRUE. To change this value, call the routine BSctx_set_guess().

void BSctx_set_guess(BSprocinfo *context, int guess);

- Convergence tolerance: The relative residual tolerance requested from the iterative solver. The default setting is $1.0 e-5$. To change this value, call the routine BSctx_set_tol().

void BSctx_set_tol(BSprocinfo *context,FLOAT tol);

If the linear system being solved is $A x=b$, the relative residual is $\|A x-b\| /\|b\|$.

- Number of RHS vectors: The number of right-hand side (RHS) vectors to be input to the linear solver. The iterative solvers in BlockSolve95 can solve for multiple RHS simultaneously. The default setting is 1 . This routine should not have to be called by the user because it is set to the correct value if the routine BSsetup_block() is called. The program example grid5 demonstrates how to solve for various numbers of RHS vectors. To change this value, call the routine BSctx_set_num_rhs().

void BSctx_set_num_rhs(BSprocinfo *context, int num_rhs);

- Preconditioner: The preconditioner to be used by the iterative solver. The choices are incomplete Cholesky (PRE_ICC), incomplete LU (PRE_ILU), SSOR (PRE_SSOR), or block Jacobi (PRE_JACOBI). The default setting is PRE_ICC. Note that the preconditioner specified must agree with the preconditioner computed. For example, if PRE_ICC is specified but an ILU preconditioner is passed to the solver, a BlockSolve95 error will be returned. To change this value, call the routine BSctx_set_pre().

void BSctx_set_pre(BSprocinfo *context, int pre);

- Iterative method: The iterative method to be used. The choices are conjugate gradients (CG), GMRES (GMRES), or SYMMLQ (SYMMLQ). The default setting is CG. To change this value, call the routine BSctx_set method().

void BSctx_set_method(BSprocinfo *context, int method); 


\section{The User Matrix Data Structure BSspmat}

To pass your matrix to BlockSolve95, you must use the matrix data structure typedef BSspmat. Given this data structure, BlockSolve95 will convert the matrix to a BlockSolve95 internal data structure of typedef BSpar mat in the routine BSmain_perm() (we discuss this routine in detail in \$5). In this section we describe two approaches for converting sparse matrix data into the BSspmat format. The first, using the routine BSeasy $A()$, is recommended and should be fairly painless starting with most standard sparse matrix storage schemes. The second, directly inserting the data and mappings into the structure BSspmat, allows for greater flexibility but is more complicated.

\subsection{The Easy Way to Convert Your Data to BSspmat}

We recommend that you use, if possible, the routine BSeasy $A()$ to generate the BSspmat data structure. The following are the essential data that you must specify for BSeasy $A()$.

- The matrix rows must be partitioned onto processors such that each processor has at least one row, each row has a unique global number, and each row is assigned to a unique processor.

- The global row numbers assigned to each processor must be contiguous. ${ }^{4}$ If processor $p$ is assigned $n_{p}$ rows, and the rows numbers start at $i_{p}$, the rows assigned to processor $p$ must be $i_{p}, i_{p}+1, \ldots, i_{p}+n_{p}-1$. For example, if there are two processors, processor 0 could have rows $0,1,2$ and processor 1 could have rows $4,5,6$ but not rows $4,6,7$. Note that the global row numbering can skip numbers; in the above example we did not use the row value 3 .

- The nonzero structure and matrix values for the rows assigned to each processor must be given in the three arrays rp, cval, and aval. The storage format for these arrays corresponds to the standard compressed row storage (CRS) scheme for sparse matrices. See pages 58-69 of [2] for a detailed discussion of various sparse storage schemes. Each processor uses the local numbering of its rows $0,1, \ldots, n_{p}-1$ to index these arrays. The first array $r p$ is an index of pointers into the two other arrays indicating where the global column numbers and nonzero values for each row begins. Thus, the $i$ th row has nonzeros in columns cval $[\operatorname{rp}[i]], \operatorname{cval}[\operatorname{rp}[i]+1], \ldots, \operatorname{cval}[\operatorname{rp}[i+1]-1]$. The nonzero values are given in corresponding locations in the array aval. The column values for each row in the array cval must be sorted from lowest to highest. Note that the entire matrix row must be represented in these arrays even if the matrix is symmetric.

Given this data, the routine BSeasy $A()$ can be called by each processor to generate the typedef BSspmat data structure. The function arguments for BSeasy $A()$ are as follows.

BSspmat *BSeasy_A(int start_num, int $\mathrm{n}$, int *rp, int *cval,

FLOAT *aval,BSprocinfo *procinfo);

Note that start_num is the starting global row number $\left(i_{p}\right.$ in the above example), and $n$ is the number of rows assigned the processor ( $n_{p}$ in the above example).

To illustrate the information BSeasy $A()$ requires, we consider the following $4 \times 4$ matrix:

$$
A=\left(\begin{array}{cccc}
a & b & 0 & 0 \\
c & d & e & 0 \\
0 & f & g & h \\
0 & 0 & i & j
\end{array}\right)
$$

\footnotetext{
${ }^{4}$ This limitation applies only to BSeasy_A(). BlockSolve95 itself does not require that row numbers be contiguous; an arbitrary row mapping can be used.
} 
Let the global numbering of the rows and columns of the matrix be 0 to 3 (e.g., $A_{12}=e$ ). Suppose that we have two processors, 0 and 1 , and that we assign rows 0 and 1 of the matrix to processor 0 and rows 2 and 3 to processor 1 . Given this distribution of the rows of the matrix and the global numbering of the columns, we would have the arrays $\mathrm{rp}$, cval, and aval as shown in Figure 2. When processor $p$ calls BSeasy_A(), its value of start_num would be given by $i_{p}$ and its value of $\mathrm{n}$ would be $n_{p}$.

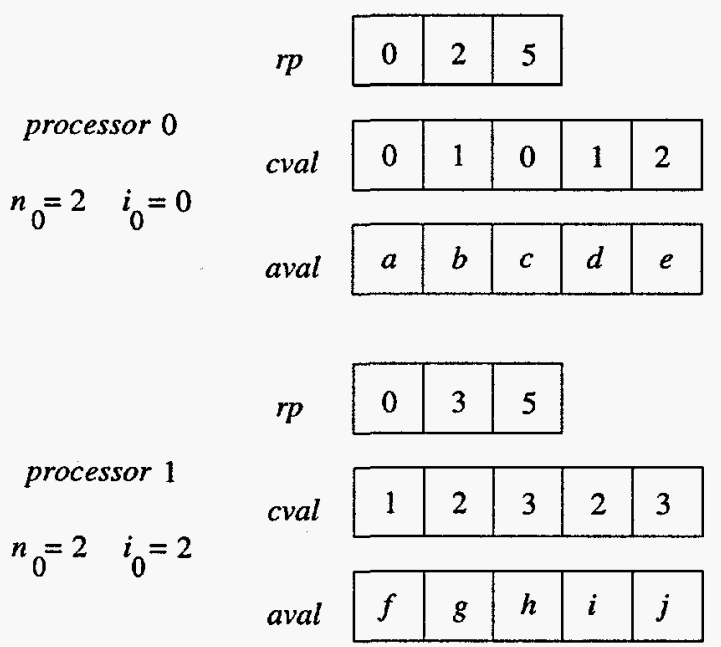

Figure 2: The values of the arrays $r p, c v a l$, and aval for the matrix given in equation 1. The matrix is partitioned so that processor 0 is assigned rows 0 and 1 and processor 1 has rows 2 and 3 . Note that indexing of the arrays and the matrices rows and columns begins with 0 .

\subsection{The Advanced Way to Convert Matrix Data to BSspmat}

You can also allocate the BSspmat without using BSeasy $A()$. Although this approach requires a bit more work, it can be more flexible. For example, we had no difficulty in writing a $\mathrm{C}$ interface routine to take a matrix written in a standard sequential format by a Fortran code and put this structure around it without duplicating the data in the Fortran sparse matrix.

Within the BSspmat data structure, each row of the matrix is represented by the structure typedef BSsprow. The data structures are specified as follows:

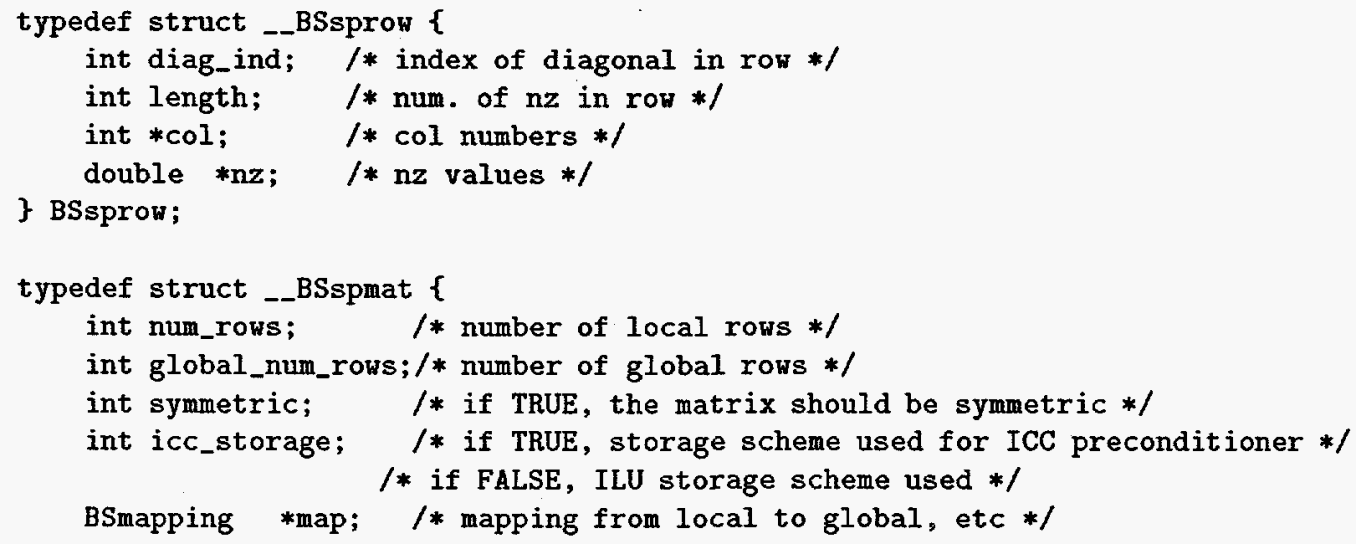




\footnotetext{
BSsprow **rows; $/ *$ the sparse rows */

\} BSspmat;
}

First, we discuss the structure BSspmat. The field num_rows contains the number of rows local to the processor. The field global_num_rows contains the total number of rows in the linear system. The fields symmetric and icc_storage respectively indicate whether the matrix is symmetric and whether an incomplete Cholesky factorization will be computed. The field map contains mapping information that will be discussed later. The field rows is an array of pointers to local rows of the sparse matrix.

In the structure BSsprow, the field diag_ind is the index of the diagonal in this row. We require that every row have a diagonal element (the value of this element could be zero). The field length contains the number of nonzero values in this row. The field col is a pointer to an array of integer values that represent the column number of each nonzero value in the row. These column numbers must be sorted in ascending order. The field $\mathbf{n z}$ is a pointer to an array of double-precision values that are the nonzero values in the row.

The mapping structure serves three purposes: (1) the mapping of local row number to global row numbers, (2) the mapping of global row numbers to local row numbers, and (3) the mapping of global row number to processor number. We provide routines for you to set up and perform this mapping (details on these routines are given in the "man" pages). You may, however, set up your own mapping and use your own routines through this data structure. The local row numbers on every processor run from 0 to num rows - 1 ; the global row numbers run from 0 to global_num_rows - 1. Each local row has a corresponding global row number.

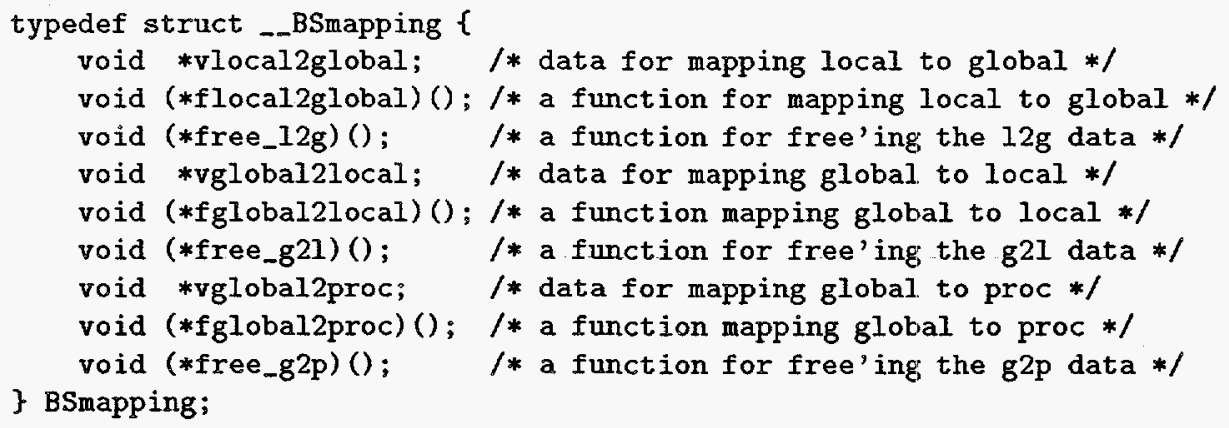

The field vlocal2global is a pointer to data that is passed into the local to global mapping function (if you are doing the mapping, you can make this point to whatever you wish). The field flocal2global is a pointer to a function for performing the local to global mapping. The field free $12 \mathrm{~g}$ is a pointer to a function for freeing the data in the field vlocal2global. The function for performing the local to global mapping takes five arguments.

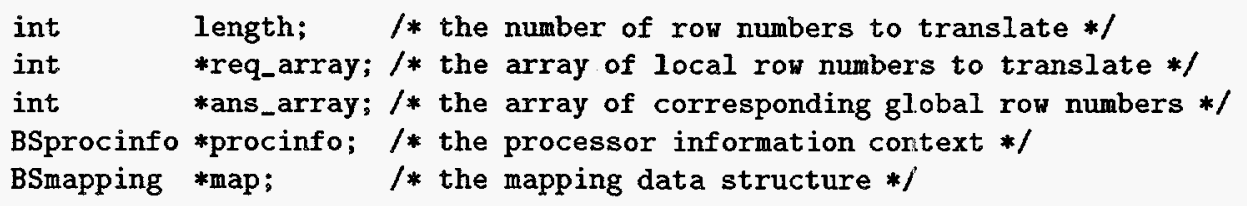

The next three fields (vglobal2local, fglobal2local, and free_g2l) are exactly the same except the mapping is from global to local row number. The mapping is performed only for rows that are local to the processor; if the mapping is attempted for a nonlocal global row number, a value of -1 is placed in the ans_array. The arguments to the mapping function are as follows. 


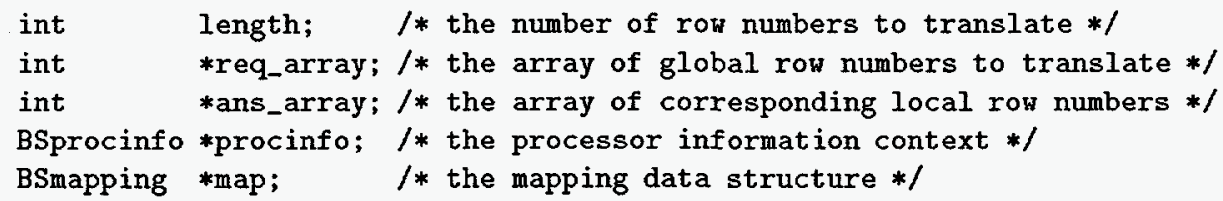

The last three fields (vglobal2proc, fglobal2proc, and free $g 2 \mathrm{p}$ ) are exactly the same except the mapping is from global row number to processor number. ${ }^{5}$ The arguments to the mapping function are as follows.

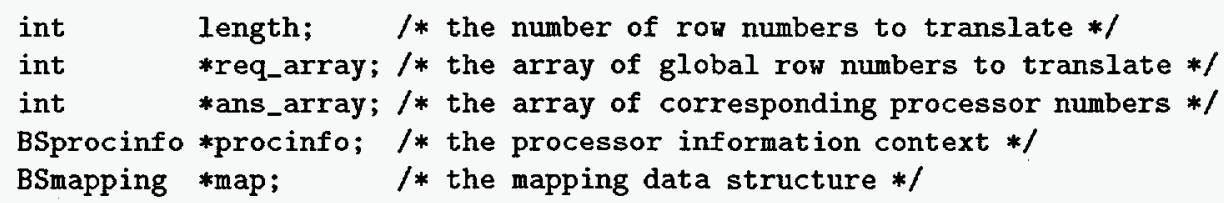

${ }^{5}$ This routine will be called by a processor for only those global row numbers that are local to that processor or for those global row numbers that are connected in the sparse matrix to rows that are local to that processor. 


\section{Manipulating and Solving Sparse Systems}

This section is divided into two parts. First, we describe how to set up the matrix and preconditioner for parallel solution. Second, we describe how to solve the linear systems after this setup has taken place.

\subsection{Manipulation and Setup}

The data structure that BlockSolve95 uses to represent sparse matrices is typedef BSpar mat. The routine that converts the user's data structure BSspmat to BSpar_mat is BSmain_perm(). This routine is called as follows.

BSpar_mat *BSmain_perm(BSprocinfo *procinfo,BSspmat *A);

This routine colors and permutes the sparse matrix to create a new version of the sparse matrix appropriate for parallel computation. A large number of options are available that are set through the context variable procinfo, as described in $\$ 3$. The user's sparse matrix is not changed permanently by this routine, but may be manipulated and restored during execution.

\subsubsection{Symmetric and Nonsymmetric Matrices}

BlockSolve95 has two versions of its internal data structure based on whether (1) the matrix nonzeros are symmetric and an incomplete Cholesky factor is to be computed, or (2) the matrix nonzeros are to be treated as if they are nonsymmetric (even if the matrix values are actually symmetric) and an incomplete ILU factor is to be computed.

The routine BSset_mat_icc_storage() must be called before BSmain_perm() to inform BlockSolve95 whether internal incomplete Cholesky storage or the incomplete LU storage is to be used. The calling sequence is

void BSset_mat_icc_storage(BSspmat *A, int storage);

where the incomplete Cholesky storage will be used if storage is TRUE, and the incomplete LU otherwise. Note that if the matrix is nonsymmetric and yet the incomplete Cholesky storage is specified, incorrect results will be obtained. BlockSolve95 assumes that the matrix is symmetric if the incomplete Cholesky option is used to minimize the required storage.

The routine BSset mat_symmetric() must be called to inform BlockSolve95 whether the matrix is symmetric (even if you use the nonsymmetric ILU internal storage format). The arguments to this routine are

void BSset_mat_symmetric(BSspmat *A, int sym);

where sym is TRUE if the matrix is symmetric and FALSE if the matrix is nonsymmetric.

In Table 3 we summarize the possible combination of options for the two functions BSset_mat_icc_storage() and BSset_mat_symmetric(). The tricky choice is whether to use ILU for a symmetric matrix. If the system is very indefinite, you may be forced to this approach to be able to compute an incomplete factor without breakdown. However, remember that this approach requires essentially twice the storage of using the incomplete Cholesky option.

\subsubsection{Fast Reorderings for Different Matrix Nonzero Values}

Often you may wish to solve more than one linear system with the same nonzero structure but different nonzero values. In this case the context should be set to "retain" important intermediate data structures by using the function BSctx_set_rt() described in §3. If BSmain_perm() 
Table 3: Consequences of choosing incomplete Cholesky or incomplete LU for symmetric or nonsymmetric matrices

\begin{tabular}{|c|c|c|}
\hline BSset_mat_symmetric() & BSset_mat_icc_storage() & Consequences \\
\hline \hline TRUE & TRUE & $\begin{array}{c}\text { Positive definite } \\
\text { preconditioner }\end{array}$ \\
\hline TRUE & FALSE & $\begin{array}{c}\text { ILU computed, twice the storage } \\
\text { of incomplete Cholesky }\end{array}$ \\
\hline FALSE & TRUE & $\begin{array}{c}\text { Incorrect } \\
\text { results }\end{array}$ \\
\hline FALSE & FALSE & $\begin{array}{c}\text { ILU computed, only } \\
\text { correct choice }\end{array}$ \\
\hline
\end{tabular}

has already been called with the "retain" parameter set to true, you can call BSmain_reperm() to permute a matrix with the same structure. The routine BSmain_reperm() is called with the following arguments.

void BSmain_reperm(BSprocinfo *procinfo,BSspmat *A,BSpar_mat *pA);

\subsubsection{Diagonal Scaling of the Linear System}

After calling BSmain_perm(), the matrix then can be scaled diagonally by calling BSscale_diag(). The function arguments are the following.

void BSscale_diag(BSpar_mat *A,FLOAT *sc_diag,BSprocinfo *procinfo);

Once the matrix has been scaled, BlockSolve95 automatically solves the scaled system

$$
\left(D^{-1} A D^{-1}\right)(D x)=\left(D^{-1} b\right)
$$

where $D$ is the diagonal matrix whose values are the square root of the absolute value of the vector sc_diag (usually chosen as the diagonal of $A$ ). Thus, if the unscaled matrix has a negative diagonal entry, it will be -1 after scaling.

\subsubsection{The Communication Data Structure BScomm}

Prior to either factoring or solving the matrix, the communication patterns used by BlockSolve95 must be created. For the factorization phase, this pattern is compiled by calling the routine BSsetupfactor().

BScomm *BSsetup_factor(BSpar_mat *A,BSprocinfo *procinfo);

For matrix solution, it is compiled by calling BSsetupforward().

BScomm *BSsetup_forward(BSpar_mat *A,BSprocinfo *procinfo);

Both routines return a communication pattern of typedef BScomm. The communication patterns may be freed by calling BSfree_comm().

void BSfree_comm(BScomm *comm_ptr); 


\subsubsection{Computing an Incomplete Factorization}

If an incomplete factor is to be computed, a copy of the matrix must be made by using the routine BScopy_par_mat().

BSpar_mat $*$ BScopy_par_mat (BSpar_mat $* A)$;

The copy is necessary because the iterative solver will require both the initial matrix and the factorization. As an aside, we note that (for the incomplete Cholesky storage scheme) the copy of the sparse matrix shares the clique storage space with the matrix from which it is copied. For more information see the man page on BScopy par_mat().

The incomplete factorization is computed by calling the routine BSfactor().

int BSfactor(BSpar_mat $* A, B S c o m m *$ comm,BSprocinfo *procinfo);

The matrix A will be overwritten with the incomplete factorization. If the factorization is successful the routine returns 0 ; otherwise a negative integer is returned whose absolute value is the row number of the color (less one) where the failure occurred. Note that the kind of factorization computed is determined by the internal representation of the matrix produced by BSmain_perm(). For example, consider the following code segment.

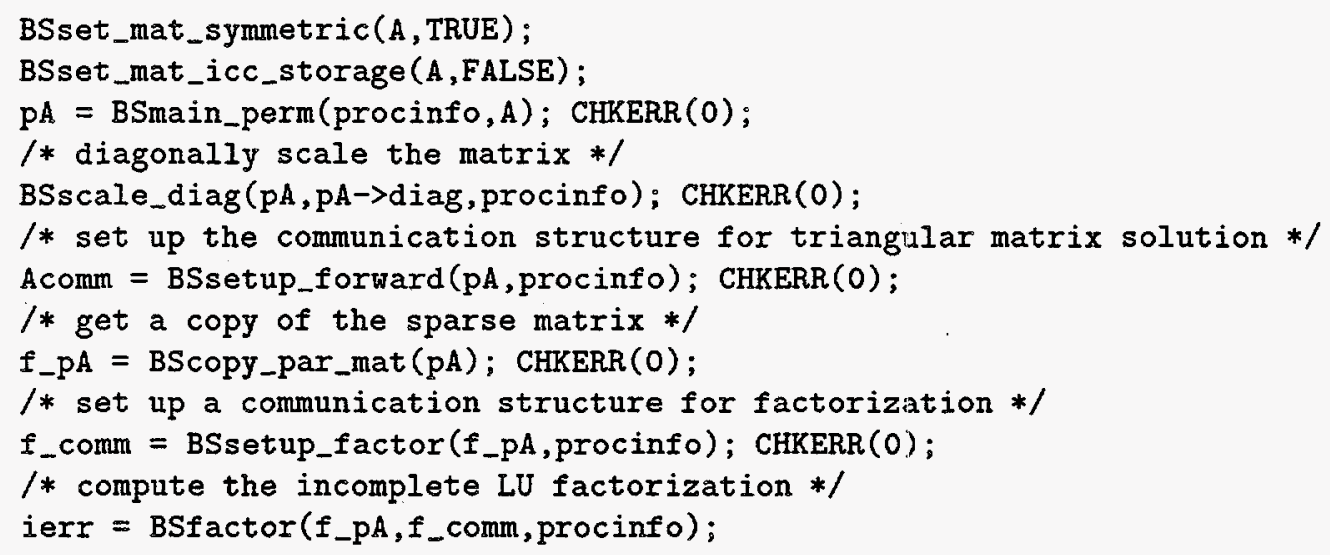

In this example the call to BSset_mat_symmetric() specifies the matrix as being symmetric. The internal storage format for the incomplete $L U$ factorization is chosen prior to the call to BSmain perm () by calling the routine BSset_mat_icc_storage(). Thus, the call to BSfactor() will compute the incomplete LU factorization rather than the incomplete Cholesky factorization even though the matrix is specified as symmetric. Also, note that the matrix has been diagonally scaled before the factorization with the call to BSscale_diag().

\subsubsection{What to Do If the Factorization Fails}

An attempt to compute the incomplete Cholesky factorization of a positive definite matrix can fail if a zero or negative diagonal is encountered during the factorization. We note that the incomplete factorization has been shown to exist only if the matrix is diagonally dominant or in several other special cases; in general, there is no guarantee that it will exist. In the case of failure the matrix must be recopied and the factorization retried. We recommend diagonally scaling the matrix and using the following loop to accomplish this task.

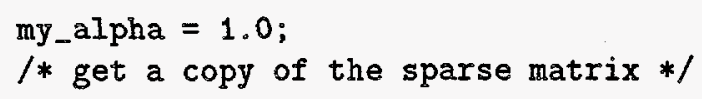




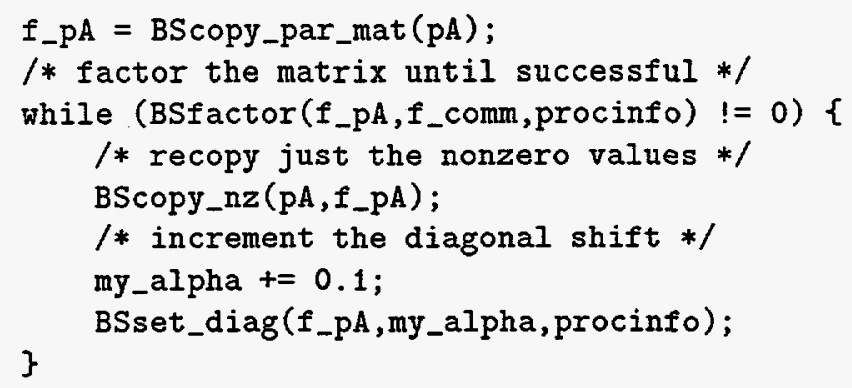

In this code segment, we are shifting the diagonal of the matrix by 0.1 every time the factorization fails. Other strategies are certainly possible and could easily be implemented by the user. The routine BSset_diag() is used to change the entire diagonal to my_alpha.

void BSset_diag(BSpar_mat *A,FLOAT my_alpha,BSprocinfo *procinfo);

\subsection{Solving the Linear System}

Once the parallel matrix and the communication structures have been created, we are ready to solve the sparse linear system. One of two routines can be called to do this: (1) BSpar_solve(), for either symmetric or nonsymmetric linear systems; and (2) BSpar_isolve(), for symmetric indefinite systems, especially those involved in "shift and invert" strategies.

BSpar_solve() can be used repeatedly to solve systems of linear equations with one or with multiple right-hand sides. The calling arguments are as follows.

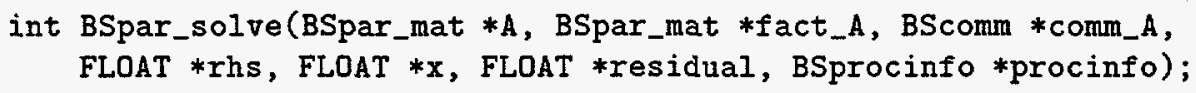

The routine returns the number of iterations taken by the solver. If the solver fails (for example, a negative curvature direction is found in a conjugate gradient iteration), the solver returns the negative of the iteration number.

The following code segment presents an example of how BSpar_solve() could be called.

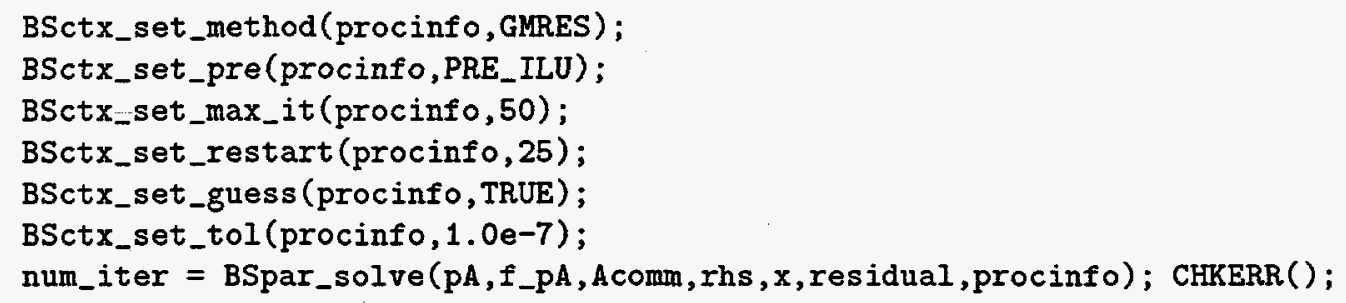

In this example the GMRES iterative method has been specified, preconditioned by an incomplete LU factorization. The preconditioner specified must agree with the preconditioner that has been computed by BSfactor(). The maximum number of iterations allowed the solver has been specified as 50, and the number of storage vector allowed GMRES before restart as 25. In this example BSctx_set_guess() has specified that the solver use an initial guess for $x$ as the zero vector. Finally, the relative residual tolerance, $\|A x-b\| /\|b\|$, has been set to 1.0e-7. Additional details on the arguments used can be found in the man page.

A second solver, BSparisolve(), is set up to solve the shifted system $(A-\sigma B) x=b$, where $A$ and $B$ are symmetric matrices, $\sigma$ is a real constant, $x$ is the solution vector, and $b$ is the right-hand side. BlockSolve95 is set up to take advantage of $B$ being NULL or $\sigma$ being zero. The calling sequence is the following. 


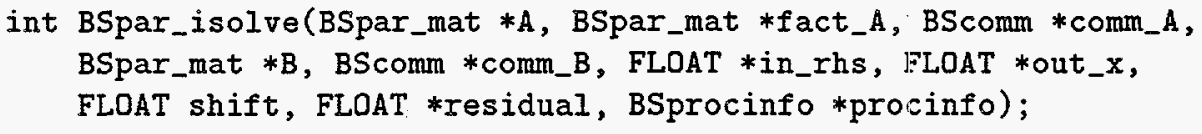

BSparisolve() uses the SYMMLQ algorithm, which requires that the preconditioner, if any, be positive definite. Symmetric diagonal scaling is not possible for an indefinite matrix, so one of the other preconditioners must be used. The restriction that the preconditioner be positive definite is too restrictive for many problems, but we know of no general-purpose alternative to SYMMLQ that takes advantage of symmetry while allowing an indefinite preconditioner. Another option would be to explicitly form $(A-\sigma B)$ and solve the resulting system with GMRES preconditioned with ILU (at the expense of twice the memory). The program examples grid7 and grid8 compare these two options. See $\S 8$ for details.

If you wish simultaneously to solve for more than one right-hand side, you must call the routine BSsetup_block () to modify the communication structure to accommodate the multiple right-hand sides. The arguments for this function are the following.

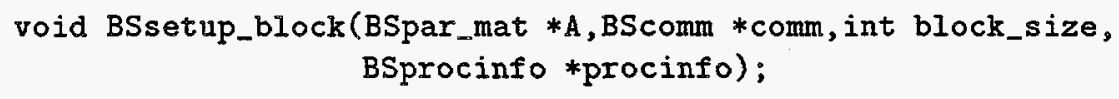

\subsection{Freeing Matrices}

To free the parallel matrix created by BSmain_perm(), call the routine BSfree_par_mat().

void BSfree_par_mat(BSpar_mat *A);

To free a copy of a parallel matrix created by BScopyparmat(), call the routine BSfree_copy_par_mat().

void BSfree_copy_par_mat (BSpar_mat *A); 


\section{Error Checking, Flop Counting, Message Passing, and the BLAS}

In this section we discuss a number of useful, miscellaneous features available in BlockSolve95. These features include error checking, flop counting, matrix size and ordering information, message-passing options, and BLAS options for optimizing processor performance.

\subsection{Error Checking within BlockSolve95}

BlockSolve95 uses an error-checking system based on the two macros SETERR() and CHKERR(), which are defined in the file include/BSdepend.h. When BlockSolve95 is compiled with the flag DEBUG_ALL defined in the file include/BSdepend.h, then when an internal error occurs (such as a failed malloc() call), BlockSolve95 returns to the user, and the error code can be checked and a traceback returned. The traceback includes the routine names and line numbers where the error occurs; this information can be useful if you suspect an error in BlockSolve95. We highly recommend the use of DEBUG_ALL until you are extremely sure of your code; even then, it is inexpensive to use DEBUG_ALL with BlockSolve95. The default is that DEBUG_ALL is set to TRUE in include/BSdepend.h.

\subsection{Flop Counting}

When the BlockSolve95 is compiled with MLOG defined, flops (floating-point operations) are being logged. (See $\$ 7$ for more information about compile-time options.) You can access the current number of flops executed by BlockSolve95 on a particular processor with the call to the function

double BSlocal_flops();

which returns a double. The total number of flops performed on all processors can be obtained by calling the function

double BSglobal_flops(BSprocinfo *procinfo);

which also returns a double.

The following code segment demonstrates how you can estimate flop rates for a section of code.

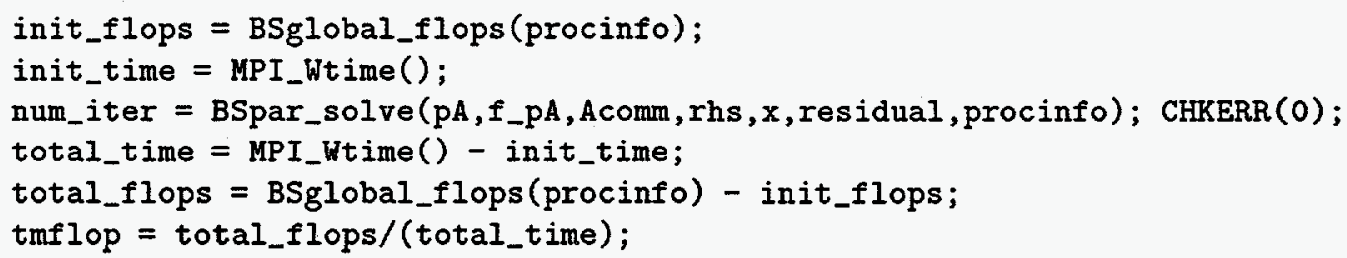

In this case, tmflop would be an estimate of the combined flop rate for all the processors involved in the call to BSpar_solve().

BlockSolve95 also does some rudimentary logging of timing and flop counts of routines within the package when compiled with MLOG defined. By calling the function

int BSprint_log(BSprocinfo *procinfo);

before the final call to BSfree_ctx(), this logging information can be printed out. 


\subsection{Matrix Statistics}

BlockSolve95 includes a number of functions that can be called to obtain information about a matrix of typedef BSpar mat. For example, the following two functions may be called to determine the matrix nonzeros locally assigned to a processor and the total (global) number of nonzeros in the matrix.

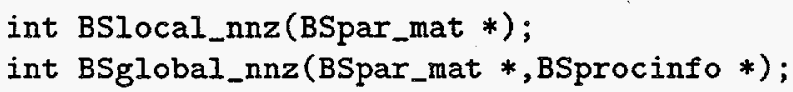

The following two functions may be called to obtain the number of i-nodes locally assigned to a processor and the global number of i-nodes.

int BSlocal_num_inodes (BSpar_mat *);

int BSglobal_num_inodes (BSpar_mat *);

The following two functions return the the number of cliques locally assigned to a processor and the global number of cliques.

int BSlocal_num_cliques(BSpar_mat *);

int BSglobal_num_cliques (BSpar_mat *);

The following function returns the number of colors used to color the graph associated with matrix clique structure.

int BSnum_colors (BSpar_mat *);

\subsection{Blocking and Nonblocking Messages}

BlockSolve95 can be compiled so that it does not use blocking (synchronous) sends and receives. (In MPI the blocking send is the function MPI_Send(); the nonblocking (asynchronous) send is the function MPI_Isend().) The MPI standard does not require that two processors simultaneously issuing blocking sends buffer messages and return; therefore deadlock can result. This problem can be avoided by using nonblocking sends, at the expense of more memory (because the message buffers cannot be freed until it is certain that the messages have been received). The IBM SP requires the use of such nonblocking sends.

BlockSolve95 compiles with the blocking or nonblocking send versions based on whether NO_BLOCKING_SEND is defined in the include file include/BSdepend.h. If you are concerned about performance or memory usage, you should experiment with the difference between blocking and nonblocking sends on your particular architecture.

\subsection{MPI Communicators and Message Number Conflicts}

BlockSolve95 currently does not make a copy of the user's MPI communicator; instead it uses as a default the current MPI_COMM_WORLD. If there is any possibility of a message-passing conflict (for example, unsatisfied wild card receives from the user's code), the communicator can be copied by using MPI_Comm_dup() and can be handed to BlockSolve95 by using BSctx_set_ps().

BlockSolve95 uses message numbers beginning at 10,000. It uses a significant but variable number of messages after that. Currently the number of messages used is $20+\left(10000^{*}\right.$ number_of_processors). The number of messages needed by BlockSolve95 depends on the problem being solved, but if the number of messages allocated to it is too small, it will detect an error and return accordingly (if DEBUG_ALL is on). The current setting of 10,000 is generous. The message numbers as well as the number of messages can be changed simply by altering include/BSprivate.h. The avoidance of conflicts in the use of message numbers can be ensured by copying the communicator as described above. 


\subsection{Inline Macros for the BLAS}

The performance of the vendor-supplied BLAS for small systems can be disappointing because of the subroutine call overhead, error checking, and special case handling (overloading) in the implementation of these BLAS. In addition, BlockSolve 95 has to gather and scatter noncontiguous vector data (although not matrix data) to use the dense BLAS. A feature in BlockSolve95 is special handling of the Level-2 BLAS for small i-node sizes; BlockSolve95 uses macros to put code inline for the special BLAS cases required. The performance improvement can be substantial. For example, in Table 4 we show the processor performance improvement obtained on the Argonne IBM SP system (RS/6000-370 processor).

Table 4: Comparison of the single-processor performance of BlockSolve95 conjugate gradient and GMRES iterations, with vendor-supplied, Level-2 BLAS (GEMV and TRMV) and the new inline macros. The symmetric systems are solved with the conjugate gradient method; the nonsymmetric systems are solved with GMRES.

\begin{tabular}{|c|c|c|c|c|}
\hline $\begin{array}{c}\text { Size of } \\
\text { i-node }\end{array}$ & $\begin{array}{c}\text { Symmetric } \\
\text { (Y or N) }\end{array}$ & $\begin{array}{c}\text { Vendor BLAS } \\
\text { (Mflops) }\end{array}$ & $\begin{array}{c}\text { BS95 Macros } \\
\text { (Mflops) }\end{array}$ & $\begin{array}{c}\text { Improvement } \\
\text { Ratio }\end{array}$ \\
\hline \hline 2 & $\mathrm{Y}$ & 3.5 & 8.3 & 2.35 \\
\hline 3 & $\mathrm{Y}$ & 6.1 & 12.5 & 2.06 \\
\hline 5 & $\mathrm{Y}$ & 10.3 & 18.3 & 1.77 \\
\hline 6 & $\mathrm{Y}$ & 14.7 & 22.3 & 1.52 \\
\hline 7 & $\mathrm{Y}$ & 17.6 & 23.2 & 1.32 \\
\hline \hline 2 & $\mathrm{~N}$ & 5.2 & 7.1 & 1.37 \\
\hline 3 & $\mathrm{~N}$ & 8.0 & 10.6 & 1.33 \\
\hline 5 & $\mathrm{~N}$ & 16.1 & 19.1 & 1.19 \\
\hline
\end{tabular}

These macros are defined in the include/BSmyblas.h. The default is that libraries are compiled with the inline versions for small i-node sizes (less than 10); otherwise, the vendorsupplied BLAS are used. These macros can be turned off by editing this file and not defining MY BLAS DTRMV_ON and MY BLAS_DGEMV_ON. Also, the maximum level of unrolling can be changed to tune performance for a particular architecture. 


\section{Installation}

Underneath the main BlockSolve95 directory are six other directories: (1) src, which contains the source code and makefiles for BlockSolve95, (2) doc, which contains the documentation for BlockSolve95, (3) examples, which contains example programs that demonstrate the use of BlockSolve95, (4) include, (5) lib, and (6) bmake.

BlockSolve95 uses the same makefile system as PETSc [1]. The README file in the main directory gives details on how to build the libraries. The makefiles will build the BlockSolve95 library in a subdirectory of the lib directory based on the machine architecture and the level of optimization. The machine architecture should be specified by the environmental variable \$PETSC_ARCH. You will have to modify the file bmake/\$PETSC_ARCH/\$PETSC_ARCH.site as directed in the README file.

Several compiler options affect BlockSolve95. The DEBUG_ALI flags were described in $\S 6$. The flag MLOG is associated with the logging facilities within BlockSolve95, and more information can be found on them in the file include/BSlog.h. A preprocessor variable called BSDOUBLE is defined in include/BSsparse.h. If BSDOUBLE is defined, BlockSolve 95 will compile a doubleprecision version; otherwise, a single-precision version is compiled. Unfortunately, the routine names for both versions are the same.

There is special case on the Cray T3D because the C code requires that FLOAT be defined as double but the BLAS and LAPACK routines require the single-precision names (although they are the double-precision versions). Details can be found in the include file include/BSsparse. $h$.

\subsection{Other Libraries}

To run BlockSolve95, you need LAPACK; the BLAS 1, 2, and 3 libraries; and an MPI implementation for the particular architecture being used. If MPI has not been installed on your system, we recommend your using MPICH. This package is available via anonymous ftp from info.mes.anl.gov in the directory pub/mpi. More information on MPI can be found on the WWW at http://ww.mes.anl.gov/mpi/index.html.

\subsection{UNIX man Pages}

UNIX man pages have been generated for the BlockSolve95 routines and are located in the directory doc/man. To use these pages you must include the path to the directory BlockSolve95/doc/man in your MANPATH environmental variable. This variable can be set with the command setenv. The tool xman provides a nice interface to these man pages. The BlockSolve 95 routines can be accessed in Section 3 and the include files in Section $h$ from the pull-down menu given by the "Sections" button on the xman toolbar.

\subsection{Availability of BlockSolve95}

The BlockSolve95 package can obtained via anonymous ftp from info.mes . anl.gov. The package is in the directory pub/BlockSolve95. In addition, BlockSolve95 is available from netlib. The current version number and last date of modification are in the file include/BSsparse.h. Please send any questions via e-mail to jones@es .utk. edu or plassmanemcs . anl .gov. Include your name, affiliation, U.S.-mail address, and e-mail address, along with a description of what you might be interested in doing with BlockSolve95.

Information on the current status of BlockSolve9.5 can be obtained on the WWW at http://ww .mcs . anl.gov/blocksolve95/index.html. 


\subsection{Differences between Previous BlockSolve Versions}

BlockSolve95 incorporates many improvements over BlockSolve v1.1 and v2.0. Among these improvements are the following.

- A compile-time option has been added that directs BlockSolve95 to use no blocking sends (see $\S 6$ or the include file include/BSdepend.h for details). This feature is necessary because certain machines (e.g., the IBM SP series architecture) have inadequate message buffering space allocated to use blocking sends.

- BlockSolve95 now uses the MPI message-passing standard instead of Chameleon for communication.

- BlockSolve95 can now compute incomplete LU factorizations for matrices that have a symmetric nonzero pattern but nonsymmetric entries. Also, the iterative solver GMRES has been added, which can be used to solve these nonsymmetric systems. See $\S 5$ for details.

- A subroutine, BSeasy $A()$, has been added that allows the user to convert a sparse matrix stored in a familiar storage format to the BlockSolve95 data structure used for parallel computation (see $\$ 4$ for details). In addition, the routine BSfree_easymat () has been added to free the data structures that BlockSolve95 allocates when BSeasy_A() is called.

- The matrix reordering routines called in BSmain_perm() have been significantly modified to greatly reduce the runtime of BlockSolve 95 for problems with only one degree of freedom per grid point. BlockSolve95 is still designed for more complex problems, but the reordering time for these simpler problems is now more satisfactory.

- The code is now ANSI-C. This should result in fewer user errors because of the type checking that can now be done on argument lists. Note that the user's code does not have to be ANSI-C to use the libraries.

- BlockSolve95 has special macros for handling the level-2 BLAS GEMV and TRMV for small i-node sizes. The performance of the vendor-supplied BLAS have proven to be inadequate in this parameter range, so inline versions of specific BLAS are now included in the include file include/BSmy_blas.h.

- The set of example programs is greatly expanded and can be used to test most aspects of BlockSolve95. See $\$ 8$ for a description of the program examples. 


\section{Example Programs}

BlockSolve95 includes a number of example programs that demonstrate most aspects of the software library. The code for these examples is contained in the examples directory.

A description of BlockSolve95 example programs, a description of the problem calling arguments is given below.

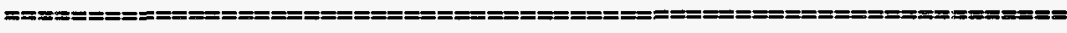

grido -- Simple example. Uses 3-d 7-point finite-difference

stencil. BlockSolve context set not to look for cliques or

inodes. Symmetric problem.

- mpirun -np P grido.ARCH PX PY PZ NX NY NZ

grid1 -- An unbalanced grid example. The processors on the left and right ends of the grid use a 7 pt stencil, those in the middle use a 27-pt stencil. BlockSolve context set not to look for cliques and inodes. Symmetric problem.

- mpirun -np P grid1.ARCH PX PY PZ NX NY NZ

grid2 -- A demonstration of running BlockSolve on two independent sets of processors simultaneously. This problem is partitioned in only the $x$ and $y$ directions. The processors on the left and right ends of the grid use a $7 \mathrm{pt}$ stencil; those in the middle use a 27-pt stencil. BlockSolve context set not to look for cliques or inodes. Symmetric problem.

- mpirun -np P grid2. ARCH PX PY NX NY NZ

grid3 -- A 2-D grid distributed across the processors. The 2-D grid is partitioned in both dimensions among the processors. The number of processors *must* be the square of an integer, e.g., 9 but not 8 . Either a 5-pt or 9=pt. stencil can be specified. BlockSolve context set not to look for cliques or inodes. Symmetric problem.

- mpirun -np P grid3.ARCH NXY NST

grid4 -- Simple example (same as grido) but this code uses lower level BlockSolve functions to set up the parallel sparse matrix data structures (done in get_mat4.c). Uses 3-d 7-point finite-difference stencil. BlockSolve context set not to look for cliques or inodes. Symmetric problem.

- mpirun -np P grid4. ARCH PX PY PZ NX NY NZ

grid5 -- Can be used to test a variety of BlockSolve features.

The grid problem is based on the 7-pt stencil; however, one can specify a number of components at each grid points. Each 
of these components will have an identical nonzero structure (inode) in the sparse matrix. One can specify either a symmetric or nonsymmetric nonzero structure. One can specify whether the BlockSolve is to look for inode/cliques or not.

. mpirun -np P grid5.ARCH PX PY PZ NX NY NZ SYM NC IN PRE METHOD SCALE NUM_RHS

grid6 -- A demonstration of setting up the BlockSolve communication structure and using the same structures to solve multiple linear systems with the same nonzero structure but different nonzero values. In this case, we compute a sequence of preconditioners by varying the diagonal shift applied to the matrix used to compute in the computation of the incomplete factor.

. mpirun -np P grid6.ARCH PX PY PZ NX NY NZ SYM IN NC

grid7 -- This example uses SYMMLQ to solve the symmetric shifted system $(A-s * B) x=b$, where $s$ is a shift and $A$ and $B$ are symmetric. (Note that this system is indefinite for large enough shift s.) Systems of this kind form the core computation in a shift and invert strategy within a Lanzcos iteration. In the usual case, $A$ would be the assembled stiffness matrix and $B$ would be the associated mass matrix. For this example, $B$ has the same nonzero structure of $A$ and its values are the absolute values of the values of $A$. Note that SYMMLQ requires that the preconditioner (the incomplete factorization of $A$ ) be positive definite!

- mpirun -np P grid7. ARCH PX PY PZ NX NY NZ PRE IN NC BMAT SHIFT

grid8 -- This example solves the same problem as that in grid7 except here we explicitly form the indefinite matrix $C=(A-s * B)$ and solve the resulting linear system system, $C x=b$, using GMRES. One can use either ILU or ICC as a preconditioner, although for large shifts the diagonal of $C$ must be shifted to avoid breakdown in the computation of the incomplete Cholesky factor.

- mpirun -np P grid8. ARCH PX PY PZ NX_NY NZ PRE_IN_NC_SHIFT

A description of possible example program arguments:

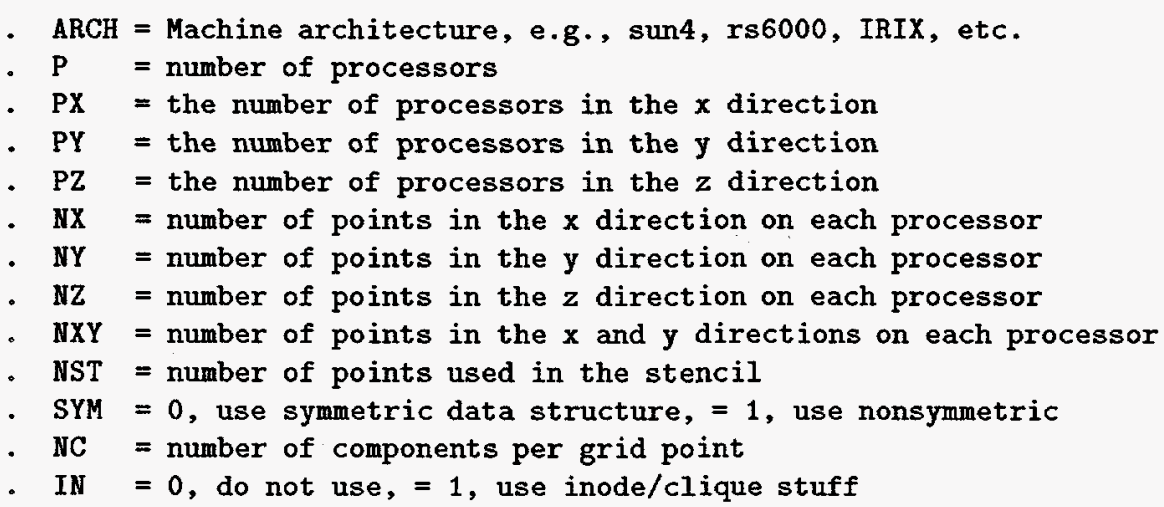


- PRE $=0$, use ICC, $=1$, use ILU

- METHOD $=0$, use CG, use GMRES

- SCALE $=0$, do not scale system, $=1$, scale system

- NUM_RHS = number of RHSs to solve for simultaneously

- $\mathrm{BMAT}=0$ = use identity for shift, 1 = use positive of $A$

- SHIFT = shift to use in the shift and invert system 


\section{Limitations and Future Plans}

The user should be aware of a few limitations in BlockSolve95:

- Each row of the matrix must have a diagonal entry. That entry may be zero, but it must be explicitly represented in the matrix structure.

- If the matrix is indefinite, one cannot solve for a block of vectors simultaneously in the current code.

- BlockSolve95 does not check for or catch exceptions associated with floating-point errors.

- Each processor involved in a BlockSolve call must have at least one row of the matrix.

- BlockSolve95 does not allow the user to mix machines with different byte orderings or different sizes for datatypes in the same computation.

Another limitation involves coloring options. It is possible with the current version that if the portion of the matrix structure contained on some processors is very different from the structure contained on other processors, then the number of colors on each of these processors can be quite different. Such a situation could arise if different-order finite elements are used on different processors (but would not arise just by applying boundary conditions to some processors, but not to others). This imbalance in the number of processors could degrade performance. A balanced coloring heuristic that addresses this situation is described in [3]; however, this heuristic is not yet included in BlockSolve9.5. 


\section{Related Software}

A number of software packages are closely related to BlockSolve95 and may be of use in particular applications.

- The software package PETSc [1] contains an interface to BlockSolve95. It can be used to access a larger number of iterative solvers, nonlinear methods, and a large number of other useful features. Information on PETSc may be obtained on the WWW at http://www.mcs.anl.gov/petsc/petsc.html.

- The SUMAA3d (Scalable Unstructured Mesh Algorithms and Applications) project is developing algorithms and software for many of the tasks associated with unstructured mesh computation. Current information about this project can be obtained on the WWW at http://www.mes .anl.gov/sumaa3d/index.html.

- Preliminary adaptive refinement software has been developed for finite element methods in two and three dimensions [12]. This software is part of the SUMAA3d project. 


\section{Acknowledgments}

We acknowledge Wing-Lok Wan for the monumental summer project of adapting the incomplete Cholesky factorization to the incomplete LU case. We thank William Gropp for his help with the MPI message-passing implementation and for his inspiration on a large number of software issues. We thank Lois Curfman McInnes, Barry Smith, and the PETSc team for the makefile facilities, motivation for conversion to ANSI-C, help with the documentation and testing, and numerous suggestions for improving the software. We also thank Satish Balay for discussions on the implementations of the required BLAS macros. 


\section{References}

[1] S. Balay, L. Curfman McInnes, W. D. Gropp, and B. F. Smith, PETSc 2.0 users manual, ANL Report ANL-95/11, Argonne National Laboratory, Argonne, Ill., Nov. 1995.

[2] R. Barrett, M. Berry, T. F. Chan, J. Demmel, J. Donato, J. Dongarra, V. EiJKHOUT, R. Pozo, C. Romine, AND H. V. DER VoRST, Templates for the Solution of Linear Systems: Building Blocks for Iterative Methods, SIAM, Philadelphia, 1994.

[3] R. K. Gjertsen, Jr., M. T. Jones, and P. E. Plassmann, Parallel heuristics for improved, balanced graph colorings, Journal of Parallel and Distributed Computing, 37 (1996), pp. 171-186.

[4] W. Gropp, E. Lusk, and A. Skjellum, Using MPI: Portable Parallel Programming with the Message Passing Interface, MIT Press, 1994.

[5] B. Hendrickson and R. Leland, The Chaco user's guide: Version 2.0, Sandia Report SAND94-2692, Sandia National Laboratories, Albuquerque, N.M., June 1995.

[6] M. T. Jones AND P. E. Plassmann, The effect of many-color orderings on the convergence of iterative methods, in Proceedings of the Copper Mountain Conference on Iterative Methods, SIAM LA-SIG, 1992.

[7] - Solution of large, sparse systems of linear equations in massively parallel applications, in Proceedings of Supercomputing '92, IEEE Computer Society Press, 1992, pp. 551560 .

[8] - Computation of equilibrium vortex structures for type-II superconductors, The International Journal of Supercomputer Applications, 7 (1993), pp. 129-143.

[9] —, A parallel graph coloring heuristic, SIAM Journal on Scientific Computing, 14 (1993), pp. 654-669.

[10] - Computational results for parallel unstructured mesh computations, Computing Systems in Engineering, 5 (1994), pp. 297-309.

[11] - Scalable iterative solution of sparse linear systems, Parallel Computing, 20 (1994), pp. $753-773$.

[12] —_, Parallel algorithms for adaptive mesh refinement, SIAM Journal on Scientific Computing, 18 (1997), pp. 686-708.

[13] Message Passing Interface Forum, MPI: A message-passing interface standard, International Journal of Supercomputing Applications, 8 (1994). 


\section{Subject Index}

adaptive refinement, 30

ANSI-C, 25

availability, 24

balanced colorings, 29

BLAS, 3, 5, 23-25

block Jacobi, 4, 11

blocking messages, 22

cliques, $4,5,10$

compiler options, 24

conjugate gradients, 4,11

convergence testing, 11

diagonal scaling, 4,10

different byte orderings, 29

directory structure, 24

error checking, 21

examples, 25,26

flop counting, 21

GMRES, 4, 11

graph coloring, 2, 6, 10

i-nodes, 10

identical nodes (i-nodes) , 4, 5, 10

incomplete Cholesky, 4, 11, 16

incomplete $\mathrm{LU}, 4,11,16$

indefinite systems, 4

inline macros, 23,25

installation, 24

Jacobi preconditioning, 11

\section{LAPACK, 24}

matrix partitioning, 4

matrix reordering, 10

memory, 2

message numbers, 22

MPI, 2

MPI communicators, 22

MPICH, 24

multiple right-hand sides, 3,11

new features, 25

nonblocking messages, 22

nonsymmetric matrix, 2, 16 parallel inner products, 4

PETSc, 24, 30

processor performance, 5

scalable performance, 3,6

single and double precision, 24

SSOR, 4,11

SUMAA3d, 30

symmetric matrix, 2, 16

SYMMLQ, 4, 11

UNIX man pages, 24

unstructured meshes, 3

WWW address, 24 


\section{Function Index}

\$PETSC_ARCH, 24

BMcomp_msg(), 6, 7

BScopy_par_mat(), 18

BScreate_ctx ()$, 8$

BSctx_print(), 9

BSctx_set_cs(), 10

BSctx_set_ct(), 10

BSctx_set_err(), 9

BSctx_set_guess(), 11, 19

BSctx_set_id(), 9

BSctx_set_is(), 10

BSctx_set_max_it(), 11

BSctx_set_method(), 11

BSctx_set_np(), 9

BSctx_set_num_rhs(), 11

BSctx_set_pr(), 9

BSctx_set_pre(), 11

BSctx_set_print_log(), 9

BSctx_set_ps(), 9, 22

BSctx_set_restart(), 11

BSctx_set_rt(), 10, 16

BSctx_set_scaling(), 10

BSctx_set_si(), 10

BSctx_set_tol(), 11

BSDOUBLE, 24

BSeasy_A(), 12, 25

BSfactor(), 18, 19

BSfinalize(), 8

BSforward(), 7

BSfree_comm(), 17

BSfree_copy_par_mat(), 20

BSfree_ctx(), 8, 21

BSfree_easymat (), 25

BSfree_par_mat(), 20

BSglobal_flops(), 21

BSglobal_nnz(), 22

BSglobal_num_cliques(), 22

BSglobal_num_inodes(), 22

BSinit(), 8

BSlocal_flops(), 21

BSlocal_nnz(), 22

BSlocal_num_cliques(), 22

BSlocal_num_inodes(), 22

BSmain_perm(), 9, 12, 25

BSmain_reperm(), 17

BSnum_colors(), 22

BSpar_isolve(), 11, 19

BSpar_solve(), 11, 19

BSprint_log(), 9, 21
BSscale_diag(), 10, 17, 18

BSset_diag(), 19

BSset_mat_icc_storage(), 16, 18

BSset_mat_symmetric(), 16, 18

BSsetup_block(), 11, 20

BSsetup_factor(), 17

BSsetup_forward(), 7, 17

CHKERR(), 21

DEBUG_ALL, 21, 24

include/BSdepend.h, 21, 22, 25

include/BSlog.h, 24

include/BSmy_blas.h, 23, 25

include/BSprivate.h, 22

include/BSsparse.h, 24

MLOG, 24

MPI_Finalize(), 8

MPI_Init(), 8

NO_BLOCKING_SEND, 22

SETERR(), 21

typedef BScomm, 17

typedef BSpar_mat, 12, 16, 22

typedef BSprocinfo, 8

typedef BSispmat, 12, 14

typedef BSisprow, 13 
Distribution for ANL-95/48

Internal:

J. M. Beumer (10)

F. Y. Fradin

G. W. Pieper

P. E. Plassmann (20)

R. L. Stevens

C. I. Wilkinson

TIS File

External:

DOE-OSTI, for distribution per UC-405 (52)

ANL-E Library

ANL-W Library

Manager, Chicago Operations Office, DOE

D. Hitchcock, DOE, Mathematical, Information, and Computational Sciences Div.

F. Howes, DOE, Mathematical, Information, and Computational Sciences Div.

M. Jones, The University of Tennessee (20)

D. Nelson, DOE, Office of Energy Research 